\title{
Pupillary changes after clinically asymptomatic high-acceleration head impacts in high school football athletes
}

\author{
Jacob R. Joseph, MD, ${ }^{1}$ Jennylee S. Swallow, MS, ${ }^{2}$ Kylene Willsey, MD, ${ }^{3}$ Andrea A. Almeida, MD, ${ }^{4}$ \\ Matthew T. Lorincz, MD, PhD, ${ }^{4}$ Robert K. Fraumann, JD, MD, ${ }^{5}$ Mark E. Oppenlander, MD, ${ }^{1}$ \\ Nicholas J. Szerlip, MD, ${ }^{1}$ and Steven P. Broglio, PhD²
}

${ }^{1}$ Department of Neurosurgery, ${ }^{2}$ Michigan Concussion Center, ${ }^{3}$ Department of Pediatrics, ${ }^{4}$ NeuroSport, and ${ }^{5}$ Department of Anesthesiology, University of Michigan, Ann Arbor, Michigan

\begin{abstract}
OBJECTIVE Previous studies have shown that clinically asymptomatic high-acceleration head impacts (HHIs) may be associated with neuronal and axonal injury, as measured by advanced imaging and biomarkers. Unfortunately, these methods of measurement are time-consuming, invasive, and costly. A quick noninvasive measurement tool is needed to aid studies of head injury and its biological impact. Quantitative pupillometry is a potential objective, rapid, noninvasive measurement tool that may be used to assess the neurological effects of HHls. In this study, the authors investigated the effect of HHls on pupillary metrics, as measured using a pupillometer, in the absence of a diagnosed concussion.

METHODS A prospective observational cohort study involving 18 high school football athletes was performed. These athletes were monitored for both the frequency and magnitude of head impacts that they sustained throughout a playing season by using the Head Impact Telemetry System. An HHI was defined as an impact exceeding $95 \mathrm{~g}$ linear acceleration and $3760 \mathrm{rad} / \mathrm{sec}^{2}$ rotational acceleration. Pupillary assessments were performed at baseline, midseason, after occurrence of an $\mathrm{HHI}$, and at the end of the season by using the NeurOptics NPi-200 pupillometer. The Sport Concussion Assessment Tool, 5th Edition (SCAT5), was also used at each time point. Comparisons of data obtained at the various time points were calculated using a repeated-measures analysis of variance and a t-test.

RESULTS Seven athletes sustained HHIs without a related diagnosed concussion. Following these HHls, the athletes demonstrated decreases in pupil dilation velocity (mean difference $0.139 \mathrm{~mm} / \mathrm{sec} ; p=0.048$ ), percent change in pupil diameter (mean difference 3.643\%; $p=0.002$ ), and maximum constriction velocity (mean difference $0.744 \mathrm{~mm} / \mathrm{sec} ; \mathrm{p}$ $=0.010$ ), compared to measurements obtained at the athletes' own midseason evaluations. No significant changes occurred between the SCAT5 subtest scores calculated at midseason and those after a high impact, although the effect sizes (Cohen's d) on individual components ranged from 0.41 to 0.65 .
\end{abstract}

CONCLUSIONS Measurable changes in pupil response were demonstrated following an $\mathrm{HHI}$. These results suggest that clinically asymptomatic HHls may affect brain reflex pathways, reflecting a biological injury previously seen when more invasive methods were applied.

https://thejns.org/doi/abs/10.3171/2019.7.JNS191272

KEYWORDS pupillometry; traumatic brain injury; concussion; accelerometry; pediatrics; trauma

$\mathrm{S}$ PORTS-RELATED concussion (SRC) is a topic of concern for many athletes, parents, and coaches, but diagnosing a concussion continues to be problematic. In addition to a concussion's complex pathophysiology, which results in a variety of clinical presentations, the diagnosis relies primarily on self-reporting of symptoms. This results in a conflict of interest on the part of the athlete with a possible brain injury, who may desire to continue playing, and depends on the athlete being adequately educated about the symptoms and repercussions

ABBREVIATIONS ANOVA = analysis of variance; CISG = Concussion in Sports Group; CTE = chronic traumatic encephalopathy; HHI = high-acceleration head impact; HITS = Head Impact Telemetry System; mTBI = mild traumatic brain injury; NPi = Neurological Pupil index; QP = quantitative pupillometry; RHI = repetitive head impact; SCAT5 = Sport Concussion Assessment Tool, 5th Edition; SRC = sports-related concussion.

SUBMITTED May 6, 2019. ACCEPTED July 26, 2019.

INCLUDE WHEN CITING Published online November 26, 2019; DOI: 10.3171/2019.7.JNS191272. 
of SRC. ${ }^{4}$ Underreporting of symptoms has been highlighted as a major issue in SRC, with research indicating that $50 \%$ of concussions are unreported..$^{14,19}$ The limitations of self-reporting of symptoms underscore the importance of developing an objective method to diagnose those athletes at risk for SRC or other brain injury.

Numerous studies have recently shown that changes in biomarkers that occur with SRC may be useful for both diagnosis determination and return-to-play decisions. ${ }^{1,31,32}$ However, biomarker analysis typically requires invasive methods of collection, advanced laboratory processing, or advanced imaging, which limits its utility in most athletic settings (e.g., high schools). Furthermore, there is now evidence of brain injury in athletes who are asymptomatic or do not otherwise seek immediate clinical attention. . $^{213,22}$ A rapid, objective, noninvasive method to identify athletes at risk and diagnose concussion would be a major advance.

The pupillary light reflex has long been a critical portion of the neurological examination. ${ }^{16}$ However, a pupillary examination typically involves use of a penlight and may involve subjective interpretation, particularly in cases in which there are subtle findings. ${ }^{23}$ More recently, use of quantitative pupillometry (QP) has become commonplace, particularly in the neurointensive care setting. ${ }^{8} \mathrm{QP}$ provides the advantage of being an objective and quantifiable biometric test, and has previously been evaluated in patients with subacute and chronic mild traumatic brain injury (mTBI). In a study in which military personnel were evaluated 15-45 days following blast-induced mTBI, clinicians found evidence of decreased dilation velocity, decreased average constriction velocity, and increased constriction latency. ${ }^{7}$ A separate study of patients who had suffered an mTBI longer than 1 year previously revealed decreases in maximum constriction velocity, average constriction velocity, average dilation velocity, maximum diameter, and constriction amplitude. ${ }^{30}$ However, QP has not been meaningfully evaluated in athletes who sustain significant head impacts. In the present study, we sought to determine if clinically asymptomatic high-acceleration head impacts (HHIs) resulted in measurable changes in neurological function, specifically changes in the pupillary light reflex as determined using QP, in the absence of other neurocognitive findings.

\section{Methods}

\section{Patient Population and Study Design}

A prospective cohort study of varsity high school football athletes was performed from July through October 2017. After approval of the study protocol had been granted by the University of Michigan Institutional Review Board (IRB), assent and written consent were obtained from all athletes and parents. The athletes and their parents were given an opportunity to provide assent and consent a second time following an IRB amendment near midseason. Any athlete who was undergoing active treatment for an mTBI, had a history of moderate or severe TBI, or had undergone neurosurgery in the past was excluded. Eighteen athletes volunteered for participation. Demographic information was collected, including each athlete's age, height, weight, concussion history, and sports participation history. All athletes underwent a preseason neurocognitive evaluation (see below) and QP. The initial assessment was performed during a period of rest, and not after an athletic event.

Each athlete's helmet was fitted with the encoder for the Head Impact Telemetry System (HITS; Riddell) to measure and record head impact data during all practices and games. All athletes were monitored for concussion symptoms by the athletic training staff, and diagnoses of SRC were made by independent physicians. Any athlete who received a diagnosis of SRC at some time during the season was excluded from the study thereafter. Repeated neurocognitive evaluation and QP were performed immediately after the athletic event during which an athlete suffered an HHI (defined as an impact that simultaneously achieved a linear acceleration of $>95 \mathrm{~g}$ and a rotational acceleration of $>3760 \mathrm{rad} / \mathrm{sec}^{2}$ ). This definition was based on data from a previous investigation in which a correction had been made to the resultant rotational acceleration value. ${ }^{5,13}$ All athletes also underwent a midseason assessment that included a neurocognitive evaluation and QP immediately after an athletic event during which the athlete did not sustain an HHI. This midseason assessment was performed approximately 2-2.5 months after the baseline assessment and took place within a 10-day period. Both the midseason and HHI assessments were performed within 1 hour of the end of a game. Finally, 1 week after the final game of the season, all athletes again underwent an additional assessment that included a neurocognitive evaluation and QP. This end-of-season evaluation was performed during a period of rest, and not after an athletic event.

\section{Neurocognitive Evaluation}

Neurocognitive evaluations were performed using the Sport Concussion Assessment Tool, 5th Edition (SCAT5). The SCAT5 is a concussion assessment tool that was designed by the Concussion in Sports Group (CISG) in 2016. ${ }^{9}$ The SCAT5 allows medical personnel to assess an athlete and report potential signs and symptoms that may help diagnose a concussion. In the current study, symptom evaluation, cognitive screening, balance examination, and delayed recall were utilized. ${ }^{9}$

\section{Quantitative Pupillometry}

Quantitative pupillometry was performed using the NPi-200 Pupillometer System (NeurOptics, Inc.). The system is used to evaluate eight parameters of the pupil. The Neurological Pupil index (NPi; NeurOptics, Inc.) value, ranging from 0 to 4.9 , determines the reactivity of the pupil. Lower values represent decreased pupillary reactivity detected by the device. The maximum size metric is defined as the maximum diameter $(\mathrm{mm})$ of the pupil, and the minimum size metric is defined as the minimum diameter (mm) of the pupil. The percent change metric is defined by the following formula: [maximum pupil diameter ( $\mathrm{mm}$ ) - minimum pupil diameter $(\mathrm{mm})] /$ maximum pupil diameter $(\mathrm{mm}) \times 100$. Constriction velocity is the rate $(\mathrm{mm} /$ sec) at which the pupil constricts after exposure to light. Latency to constriction is the amount of time (seconds) 
TABLE 1. Comparison of QP results obtained following HHIs and at midseason

\begin{tabular}{lccc}
\hline & $\begin{array}{c}\mathrm{HHI} \\
\text { Evaluation }\end{array}$ & $\begin{array}{c}\text { Midseason } \\
\text { Evaluation }\end{array}$ & $\begin{array}{c}\mathrm{p} \\
\text { Value }\end{array}$ \\
\hline NPi value & $3.721 \pm 0.423$ & $3.936 \pm 0.308$ & 0.069 \\
\hline Max diameter, mm & $5.163 \pm 0.698$ & $5.504 \pm 0.716$ & 0.178 \\
\hline Min diameter, mm & $3.599 \pm 0.554$ & $3.648 \pm 0.520$ & 0.775 \\
\hline $\begin{array}{l}\text { \% change in pupil } \\
\text { diameter }\end{array}$ & $30.286 \pm 2.701$ & $33.929 \pm 2.269$ & 0.002 \\
\hline $\begin{array}{c}\text { Max constriction velocity, } \\
\text { mm/sec }\end{array}$ & $4.382 \pm 0.654$ & $5.126 \pm 0.534$ & 0.010 \\
\hline $\begin{array}{c}\text { Latency to constriction, } \\
\text { sec }\end{array}$ & $0.228 \pm 0.032$ & $0.213 \pm 0.019$ & 0.166 \\
\hline $\begin{array}{c}\text { Constriction velocity, } \\
\text { mm/sec }\end{array}$ & $3.121 \pm 0.573$ & $3.429 \pm 0.378$ & 0.082 \\
\hline Dilation velocity, mm/sec & $1.359 \pm 0.290$ & $1.498 \pm 0.261$ & 0.048 \\
\hline
\end{tabular}

Values are expressed as means \pm SDs. Boldface type indicates statistical significance.

between the eye's exposure to a light stimulus and initiation of pupil constriction. Maximum constriction velocity is the top rate $(\mathrm{mm} / \mathrm{sec})$ at which the pupil constricts. Dilation velocity is the rate $(\mathrm{mm} / \mathrm{sec})$ at which the pupil dilates after constriction. Testing takes less than 2 minutes and was conducted in similar ambient lighting throughout the study.

\section{Statistical Methods}

Means and standard deviations of values representing the athletes' demographic information, including age, height, weight, years participating in football, and previous concussion, were calculated using descriptive statistics. Comparisons were made between the baseline, midpoint, and end-of-season measurements obtained for athletes who completed all time points, so that we could evaluate changes in QP and neurocognitive assessments over the course of the season. Additionally, comparisons were made between changes in the results of the neurocognitive and QP assessments after HHI and at the midpoint evaluation by using a within-subject control group. The midpoint evaluation was used as a comparator to minimize the effect of game play itself. A repeated-measures analysis was used to evaluate the F-statistic and the significance of neurocognitive scores across the season in all athletes at the baseline, midpoint, and end-of-season evaluations. Neurocognitive scores at the midpoint evaluation were compared with those obtained immediately following the HHI evaluation by using a paired t-test, by which we calculated the mean, standard deviation, significance, and effect size (Cohen's d). A two-way (left/right eye by time) analysis of variance (ANOVA) with repeated measures was used to examine the pupil characteristics at the baseline, midpoint, and end-of-season evaluations, as well as between the midpoint and HHI evaluations. Descriptive statistics, $95 \%$ confidence intervals, significance ( $\mathrm{p}$ values), and pairwise comparisons were analyzed for each repeated-measures ANOVA when significant main effects had been identified. Statistical significance was noted when $\mathrm{p}<0.05$. All analyses were performed using IBM SPSS Statistics 24.0 software (IBM Corp.).

\section{Results}

Of the 18 enrolled athletes, five athletes were excluded from the analyses: one athlete withdrew from the study, two athletes sustained orthopedic injuries, and two athletes did not complete the second assent-consent process. The remaining group of 13 athletes were $17.2 \pm 0.6$ years old and $180.9 \pm 6.2 \mathrm{~cm}$ tall, weighed $87.5 \pm 18.9 \mathrm{~kg}$, and had participated in $6.15 \pm 2.70$ years of football at the time of enrollment. Eight athletes (61.5\%) had sustained at least one concussion prior to the start of the study, with five $(38.5 \%)$ of these athletes having sustained two previous concussions. A total of 49 athletic events, including 37 practices and 12 games, were observed, capturing a total of 7045 head impacts.

Seven athletes (53.8\%) experienced an $\mathrm{HHI}$ at some time during the course of the season. Of these, six athletes experienced an HHI prior to the midpoint evaluation. On average, the athletes sustained $334.71 \pm 269.33$ impacts prior to meeting the threshold for an HHI, which averaged $114.01 \pm 13.07 \mathrm{~g}$ linear acceleration and 5298.41 $\pm 672.85 \mathrm{rad} / \mathrm{sec}^{2}$ rotational acceleration. Midseason testing occurred after an average of $555.43 \pm 346.10$ impacts. The mean maximum head impact on the day of midseason testing was $50.60 \pm 27.89 g$ linear acceleration and 1877.25 $\pm 1183.48 \mathrm{rad} / \mathrm{sec}^{2}$ rotational acceleration.

When we used the midpoint evaluation as a within-subject control, the QP revealed significant decreases in dilation velocity (mean difference $0.139 \mathrm{~mm} / \mathrm{sec} ; \mathrm{p}=0.048$ ), percent change in pupil diameter (mean difference 3.643; $\mathrm{p}=0.002$ ), and maximum constriction velocity (mean difference $0.744 \mathrm{~mm} / \mathrm{sec} ; \mathrm{p}=0.010$ ) at the assessment made after HHI when compared to values at the athletes' own midpoint evaluations (Table 1). There was no significant difference in the NPi, maximum size metric (maximum diameter), minimum size metric (minimum diameter), latency to constriction, or constriction velocity (all $\mathrm{p}>$ 0.05 ) at the same time point. An evaluation of the SCAT5 components did not reveal any differences between values obtained at the HHI evaluation and those obtained at the midpoint evaluation $(p>0.05)$. The associated data are summarized in Table 2.

When we compared differences in the QP over the course of the season, there was a significant change in both constriction velocity $(\mathrm{p}=0.019)$ and maximum constriction velocity $(\mathrm{p}=0.043$; Table 3$)$. Neurocognitive testing performed using the SCAT5 did not show any significant differences over the course of the season. The associated data are summarized in Table 4.

\section{Discussion}

Concussion was first defined by the Congress of Neurological Surgeons in 1966 as

a clinical syndrome characterized by immediate and transient impairment of neural function, such as alteration of consciousness, disturbance of vision, equilibrium, etc., due to mechanical forces. ${ }^{11}$ 
TABLE 2. Neurocognitive performance following $\mathrm{HHI}$ and at midseason

\begin{tabular}{lcccc}
\hline \multicolumn{1}{c}{ Neurocognitive Testing* } & HHI Evaluation & Midseason Evaluation & $p$ Value & Effect Size \\
\hline Self-report: symptom severity & $10 \pm 18.64$ & $6 \pm 8.39$ & 0.767 & -0.127 \\
\hline Self-report: no. of symptoms & $3.67 \pm 5.61$ & $3.67 \pm 4.13$ & 1 & 0 \\
\hline Self-report: \% of normal & $87.5 \pm 11.73$ & $95.83 \pm 8.01$ & 0.175 & 0.645 \\
\hline Orientation & $4.33 \pm 1.21$ & $4.86 \pm 0.378$ & 0.203 & 0.598 \\
\hline Immediate recall & $14.5 \pm 1.22$ & $14.71 \pm 0.488$ & 0.363 & 0.408 \\
\hline Delayed recall & $2.67 \pm 1.21$ & $3.57 \pm 1.51$ & 0.363 & 0.408 \\
\hline Concentration & $3.17 \pm 1.33$ & $3.43 \pm 0.786$ & 0.465 & 0.323 \\
\hline Balance & $12 \pm 5.37$ & $10.43 \pm 3.87$ & 0.605 & -0.225 \\
\hline
\end{tabular}

Values are expressed as means \pm SDs

* Neurocognitive testing was performed using the SCAT5. ${ }^{9}$

Since that time the definition of SRC has become more varied and complex. A recent systematic review identified 14 articles that provided definitions of concussion by six different organizations. ${ }^{20}$ The authors of the review recommended that the clinical definition of concussion include elements of biomechanics, physiology, clinical findings, neuroimaging, and fluid biomarkers/genetics. These recommendations culminated in a definition of SRC provided by the CISG in 2016 as

a traumatic brain injury induced by biomechanical forces... [that] typically results in the rapid onset of short-lived impairment of neurological function... [and] results in a range of clinical signs and symptoms. ${ }^{21}$

Because of the ambiguity in reporting and identifying the subjective clinical symptoms of SRC, the definition would be improved by an objective measure for diagnosis. ${ }^{4}$

In the present study, there was evidence of biometric changes, as measured by the pupillary light reflex assessed after clinically asymptomatic HHI. Relative to values obtained at the participant's own evaluation following an uneventful (no HHI) athletic event, there were significant decreases in dilation velocity, percent change of pupil diameter, and maximum constriction velocity. No significant differences were seen in the results of neurocognitive assessments that were performed after the HHI and nonHHI evaluations. These results indicate that the forces experienced by these athletes were sufficient to alter intrinsic brain reflex pathways, even when the effects of the HHI were otherwise clinically silent. Clinically asymptomatic HHIs have already been associated with increases in serum tau and UCH-L1, both biomarkers of TBI. ${ }^{13}$ Coupled with the QP data, an otherwise asymptomatic HHI potentially meets the criteria proposed for concussion by the CISG based on an objective biomechanical, physiological, and biomarker evaluation. Previous literature has suggested that there is a wide spectrum of mTBIs. ${ }^{18}$ The results reported in the present study indicate that an HHI may be another grade on the concussion spectrum that cannot be captured by standard clinical testing.

Concussions, as clinically defined, have remained in the spotlight over the last 10 years due to ongoing concern about their relationship to chronic traumatic encephalopathy (CTE). While the prevailing theories are that CTE develops as a result of repetitive head impacts (RHIs), the exact cause and mechanism of CTE is still under investigation. ${ }^{27} \mathrm{In}$ fact, there is evidence against the prevailing understanding of RHIs as the sole mechanism of CTE ${ }^{17,26}$ In the present study, HHI was associated with pupillary changes that were not seen during evaluations made following events during which there were no HHIs. These results suggest that HHIs may have a more profound impact on the health of athletes than a typical collision. RHIs, which by definition entail $100 \%$ of head impacts, constitute a nearly impossible entity to avoid short of abandoning all collision sports. However, an HHI accounts for only $0.01 \%$ of all head impacts experienced by high school athletes. ${ }^{13}$ Given this low incidence, an HHI

TABLE 3. Comparison of QP results over the course of the season

\begin{tabular}{lccrcc}
\hline & Baseline Evaluation & Midseason Evaluation & End-of-Season Evaluation & F-Test & $p$ Value \\
\hline NPi value & $3.808 \pm 0.401$ & $3.981 \pm 0.343$ & $3.919 \pm 0.290$ & 1.501 & 0.265 \\
\hline Max diameter, $\mathrm{mm}$ & $5.258 \pm 0.931$ & $5.341 \pm 0.658$ & $5.290 \pm 0.808$ & 0.038 & 0.962 \\
\hline Min diameter, $\mathrm{mm}$ & $3.580 \pm 0.558$ & $3.513 \pm 0.513$ & $3.550 \pm 0.493$ & 0.061 & 0.941 \\
\hline$\%$ change & $31.231 \pm 6.849$ & $34.385 \pm 3.499$ & $32.769 \pm 3.861$ & 2.751 & 0.107 \\
\hline Max constriction velocity, $\mathrm{mm} / \mathrm{sec}$ & $4.576 \pm 1.247$ & $5.165 \pm 0.596$ & $4.744 \pm 0.712$ & 4.264 & 0.043 \\
\hline Latency to constriction, sec & $0.215 \pm 0.031$ & $0.220 \pm 0.026$ & $0.232 \pm 0.031$ & 2.511 & 0.126 \\
\hline Constriction velocity, $\mathrm{mm} / \mathrm{sec}$ & $2.874 \pm 0.923$ & $3.467 \pm 0.457$ & $3.222 \pm 0.481$ & 5.788 & 0.019 \\
\hline Dilation velocity, $\mathrm{mm} / \mathrm{sec}$ & $1.170 \pm 0.470$ & $1.477 \pm 0.219$ & $1.284 \pm 0.309$ & 4.829 & 0.068 \\
\hline
\end{tabular}

Values are expressed as means \pm SDs. Boldface type indicates statistical significance. 
TABLE 4. Neurocognitive performance in all athletes over the course of the season

\begin{tabular}{|c|c|c|c|c|c|}
\hline Neurocognitive Testing* & Baseline Evaluation & Midseason Evaluation & End-of-Season Evaluation & F-Test & p Value \\
\hline Self-report: symptom severity & $5.23 \pm 5.42$ & $5.38 \pm 7.65$ & $2.0 \pm 2.89$ & 3.149 & 0.083 \\
\hline Self-report: no. of symptoms & $3.23 \pm 3.24$ & $2.77 \pm 3.56$ & $1.54 \pm 2.18$ & 3.069 & 0.087 \\
\hline Self-report: $\%$ of normal & $92.31 \pm 9.61$ & $96.77 \pm 6.57$ & $95.77 \pm 7.32$ & 0.733 & 0.502 \\
\hline Orientation & $4.69 \pm 0.48$ & $4.69 \pm 0.48$ & $4.92 \pm 0.28$ & 2.912 & 0.097 \\
\hline Immediate recall & $14.08 \pm 1.8$ & $14.77 \pm 0.44$ & $14.62 \pm 0.77$ & 0.992 & 0.402 \\
\hline Delayed recall & $3.08 \pm 1.49$ & $3.54 \pm 1.33$ & $3.15 \pm 1.63$ & 1.413 & 0.284 \\
\hline Concentration & $3.31 \pm 1.32$ & $3.31 \pm 0.75$ & $3.15 \pm 1.57$ & 0.183 & 0.835 \\
\hline Balance & $12.69 \pm 6.86$ & $10.69 \pm 3.3$ & $10.77 \pm 5.97$ & 0.83 & 0.462 \\
\hline
\end{tabular}

Values are expressed as means \pm SDs.

${ }^{*}$ Neurocognitive testing was performed using the SCAT5. ${ }^{9}$

appears to be an actionable entity. Previous studies have suggested that the frequency of high-speed collisions can be regulated out of football and, perhaps, other sports. Potential interventions include an increased football field width to decrease the likelihood of collisions, fewer practices involving contact, rule changes such as targeting penalties, and coaching interventions into improper tackling techniques. ${ }^{6,10,12,29}$

A clear causal relationship between chronic neurodegenerative disease and collision sports has yet to be established, in part due to the difficulty of defining and documenting all head impact and head injury events. Future studies of head impacts and their sequelae would be improved by the development of a reproducible definition of head injury that does not rely on clinical acumen. Helmet accelerometry is one method by which these impacts can be quantified, although it is limited in its ability to correlate impact with injury. ${ }^{24}$ Based on the results seen here, QP may be a rapid noninvasive method of measuring brain injury in conjunction with or without helmet accelerometry. With tools such as QP, as well as others such as eye-tracking devices, a better objective understanding of the relationship between impact and injury (both shortterm and long-term) is possible. ${ }^{3,25,28}$ Furthermore, simple devices such as these can enhance the detection of head injury on a larger scale, benefitting the many youth sports that do not have access to athletic trainers. ${ }^{15}$ These objective measures will allow more rigorous studies to be performed on the relationship between head impacts and their sequelae.

There were a number of limitations in this study. The sample size was low, increasing the effect of outliers. The multiplicity of hypotheses tested here further worsened the effect of the low sample size and could potentially have altered the results. A significantly larger cohort is needed to elucidate the changes that occur following an HHI. In addition, the results may have been affected by the use of within-subject controls rather than a separate cohort. The clinical implications of the subtle pupillary change seen with QP are also unclear.

\section{Conclusions}

Measurable changes in pupil response were demonstrated following HHIs. Based on these results, we can infer that nonconcussive HHIs may affect intrinsic brain reflex pathways and may reflect biological injuries that previously were found using more invasive methods.

\section{References}

1. Asken BM, Bauer RM, DeKosky ST, Svingos AM, Hromas G, Boone JK, et al: Concussion BASICS III: serum biomarker changes following sport-related concussion. Neurology 91:e2133-e2143, 2018

2. Bailes JE, Petraglia AL, Omalu BI, Nauman E, Talavage T: Role of subconcussion in repetitive mild traumatic brain injury. J Neurosurg 119:1235-1245, 2013

3. Bin Zahid A, Hubbard ME, Lockyer J, Podolak O, Dammavalam VM, Grady M, et al: Eye tracking as a biomarker for concussion in children. Clin J Sport Med [epub ahead of print], 2018

4. Broglio SP, Guskiewicz KM, Norwig J: If you're not measuring, you're guessing: the advent of objective concussion assessments. J Athl Train 52:160-166, 2017

5. Broglio SP, Schnebel B, Sosnoff JJ, Shin S, Fend X, He X, et al: Biomechanical properties of concussions in high school football. Med Sci Sports Exerc 42:2064-2071, 2010

6. Broglio SP, Williams RM, O’Connor KL, Goldstick J: Football players' head-impact exposure after limiting of fullcontact practices. J Athl Train 51:511-518, 2016

7. Capó-Aponte JE, Urosevich TG, Walsh DV, Temme LA, Tarbett AK: Pupillary light reflex as an objective biomarker for early identification of blast-induced mTBI. J Spine [epub ahead of print], 2013

8. Couret D, Boumaza D, Grisotto C, Triglia T, Pellegrini L, Ocquidant $\mathrm{P}$, et al: Reliability of standard pupillometry practice in neurocritical care: an observational, double-blinded study. Crit Care 20:99, 2016

9. Echemendia RJ, Meeuwisse W, McCrory P, Davis GA, Putukian M, Leddy J, et al: The Sport Concussion Assessment Tool 5th Edition (SCAT5): background and rationale. Br J Sports Med 51:848-850, 2017

10. Emery CA, Black AM, Kolstad A, Martinez G, Nettel-Aguirre A, Engebretsen L, et al: What strategies can be used to effectively reduce the risk of concussion in sport? A systematic review. Br J Sports Med 51:978-984, 2017

11. Gurdjian E, Volis H: Report of Ad Hoc Committee to Study Head Injury Nomenclature. Neurosurgery 12 (CN Suppl 1):386-394, 1966

12. Joseph JR, Khalsa SS, Smith BW, Park P: Impact of increased football field width on player high-speed collision rate. World Neurosurg 103:73-77, 2017

13. Joseph JR, Swallow JS, Willsey K, Lapointe AP, Khalatbari S, Korley FK, et al: Elevated markers of brain injury 
as a result of clinically asymptomatic high-acceleration head impacts in high-school football athletes. J Neurosurg 130:1409-1788, 2019

14. Kroshus E, Garnett B, Hawrilenko M, Baugh CM, Calzo JP: Concussion under-reporting and pressure from coaches, teammates, fans, and parents. Soc Sci Med 134:66-75, 2015

15. Kroshus E, Rivara FP, Whitlock KB, Herring SA, Chrisman SPD: Disparities in athletic trainer staffing in secondary school sport: implications for concussion identification. Clin J Sport Med 27:542-547, 2017

16. Larson MD, Singh V: Portable infrared pupillometry in critical care. Crit Care 20:161, 2016

17. Lye TC, Shores EA: Traumatic brain injury as a risk factor for Alzheimer's disease: a review. Neuropsychol Rev 10:115-129, 2000

18. Mayer AR, Quinn DK, Master CL: The spectrum of mild traumatic brain injury: a review. Neurology 89:623-632, 2017

19. McCrea M, Hammeke T, Olsen G, Leo P, Guskiewicz K: Unreported concussion in high school football players: implications for prevention. Clin J Sport Med 14:13-17, 2004

20. McCrory P, Feddermann-Demont N, Dvořák J, Cassidy JD, McIntosh A, Vos PE, et al: What is the definition of sportsrelated concussion: a systematic review. Br J Sports Med 51:877-887, 2017

21. McCrory P, Meeuwisse W, Dvořák J, Aubry M, Bailes J, Broglio S, et al: Consensus statement on concussion in sport-the 5 th International Conference on Concussion in Sport held in Berlin, October 2016. Br J Sports Med 51:838-847, 2017

22. Nauman EA, Talavage TM: Subconcussive trauma. Handb Clin Neurol 158:245-255, 2018

23. Olson DM, Stutzman S, Saju C, Wilson M, Zhao W, Aiyagari $\mathrm{V}$ : Interrater reliability of pupillary assessments. Neurocrit Care 24:251-257, 2016

24. Rowson B, Tyson A, Rowson S, Duma S: Measuring head impacts: accelerometers and other sensors. Handb Clin Neurol 158:235-243, 2018

25. Samadani U, Li M, Qian M, Laska E, Ritlop R, Kolecki R, et al: Sensitivity and specificity of an eye movement trackingbased biomarker for concussion. Concussion 1:CNC3, 2015

26. Smith DH, Johnson VE, Stewart W: Chronic neuropathologies of single and repetitive TBI: substrates of dementia? Nat Rev Neurol 9:211-221, 2013

27. Smith DH, Johnson VE, Trojanowski JQ, Stewart W: Chronic traumatic encephalopathy-confusion and controversies. Nat Rev Neurol 15:179-183, 2019

28. Sussman ES, Ho AL, Pendharkar AV, Ghajar J: Clinical evaluation of concussion: the evolving role of oculomotor assessments. Neurosurg Focus 40(4):E7, 2016

29. Swartz EE, Broglio SP, Cook SB, Cantu RC, Ferrara MS, Guskiewicz KM, et al: Early results of a helmetless-tackling intervention to decrease head impacts in football players. J Athl Train 50:1219-1222, 2015

30. Thiagarajan P, Ciuffreda KJ: Pupillary responses to light in chronic non-blast-induced mTBI. Brain Inj 29:1420-1425, 2015

31. Zetterberg H, Blennow K: Fluid biomarkers for mild traumatic brain injury and related conditions. Nat Rev Neurol 12:563-574, 2016

32. Zetterberg H, Winblad B, Bernick C, Yaffe K, Majdan M, Johansson G, et al: Head trauma in sports-clinical characteristics, epidemiology and biomarkers. J Intern Med 285:624-634, 2019

\section{Disclosures}

Dr. Joseph reports having received support for the study from NeurOptics, Inc., and the Blue Cross Blue Shield Foundation of Michigan. Dr. Oppenlander is a consultant for Globus Medical, DePuy Synthes, and LifeNet Health.

\section{Author Contributions}

Conception and design: Joseph, Swallow, Szerlip, Broglio. Acquisition of data: Joseph, Swallow, Willsey, Almeida, Lorincz. Analysis and interpretation of data: Joseph, Swallow, Willsey, Szerlip, Broglio. Drafting the article: Joseph, Swallow, Willsey, Szerlip, Broglio. Critically revising the article: Joseph, Swallow, Almeida, Lorincz, Fraumann, Oppenlander, Szerlip, Broglio. Reviewed submitted version of manuscript: all authors. Approved the final version of the manuscript on behalf of all authors: Joseph. Statistical analysis: Joseph, Swallow, Broglio. Administrative/technical/ material support: Joseph, Broglio. Study supervision: Joseph, Szerlip, Broglio.

\section{Correspondence}

Jacob R. Joseph: University of Michigan, Ann Arbor, MI. jrjosephmd1@gmail.com. 